\title{
SENSITIVE AMPEROMETRIC NANOBIOSENSOR FOR DETECTION OF SARCOSINE - POTENTIAL PROSTATE CANCER MARKER - IN URINE SAMPLES
}

\author{
Štefánia HRONČEKOVÁ, Tomáš BERTÓK, Ján TKÁČ \\ Slovak Academy of Sciences, Institute of chemistry, Bratislava, Slovakia, EU, \\ chemshrn@savba.sk
}

https://doi.org/10.37904/nanocon.2021.4313

\begin{abstract}
In this work, two-dimensional layered material $\mathrm{Ti}_{3} \mathrm{C}_{2} \mathrm{~T} \times \mathrm{MXene}$ has been used to immobilize enzyme sarcosine oxidase to fabricate a nanostructured biosensor. The device was applied for detection of sarcosine, a potential prostate cancer biomarker, in urine samples for the first time. The morphology and structures of MXene have been characterised by atomic force microscopy (AFM) and scanning electron microscopy (SEM). Electrochemical, SEM and AFM results revealed that MXene along with chitosan is a good candidate for enzyme immobilization to fabricate a sensitive nanobiosensor. The prepared device exhibited a good analytical performance with a low detection limit of $18 \mathrm{nM}$ and linear range from 0.09 to $7.8 \mu \mathrm{M}$. The proposed biosensing method also demonstrated high recovery index for sarcosine detection in urine samples in clinically relevant range $(102.6 \%)$.
\end{abstract}

Keywords: MXene, sarcosine, biosensor, prostate cancer

\section{INTRODUCTION}

Cancer is now widely recognized as a global problem that unfortunately lacks a global solution. Prostate cancer $(\mathrm{PCa})$ is the most diagnosed cancer type among men and one of the most prevalent cancer types in general. It is a leading cause of deaths among men with an incidence of 1.1 million cases a year resulting in 366,000 deaths annually [1]. The gold standard in early-stage PCa diagnostics/screening is analysis of the level of prostate-specific antigen (PSA) in blood serum. PSA is a glycoprotein produced by the prostate gland which is responsible for seminal fluid liquefaction. Although widely used in connection with cancer, elevated levels of PSA will identify prostate disease, but not necessarily PCa. Hence, PSA is rather prostate-specific than cancerspecific and should not be used as reliable PCa diagnostic biomarker [2]. The key to effective prostate cancer management is early and accurate diagnostics, which includes the ability to differentiate between men with aggressive disease that require immediate treatment and those with slow-growing cancer that may not cause any significant difficulties within their lifetime. Utilizing a panel of diverse biomarkers is the most appropriate method. Potential PCa biomarkers include sarcosine, zinc-alpha-2-glycoprotein, prostate cancer antigen 3 , Cofilin-1, annexin A3 as well as transmembrane protease serine 2-ETS-related gene [3,4]. This work is focused on sensitive electrochemical detection of sarcosine, which can provide significant discrimination between $\mathrm{PCa}$ patients and non-cancer individuals, with an area under curve characteristic (AUC) of 0.833 [5]. Sarcosine, also known as $\mathrm{N}$-methylglycine, is a non-proteinogenic amino acid naturally occurring in muscles and various other body tissues [6]. Although the measurements in blood have been reported as unremarkable, significantly elevated levels in urine have been recorded in some studies, for patients with PCa. Normally, the level of sarcosine in the urine of the PCa patients is very low, approximately $20 \mathrm{nM}$ to $5 \mu \mathrm{M}$ [7]. This work describes construction of a novel sensitive sarcosine biosensor based on immobilization of sarcosine oxidase (SOx, EC 1.5.3.1) on the surface of pre-treated glassy carbon electrodes (GCEs). Two-dimensional layered nanomaterial $\mathrm{Ti}_{3} \mathrm{C}_{2} \mathrm{~T}_{\mathrm{X}}$ (a member of the MXene family) was used to immobilise enzyme SOx to fabricate a nanostructured biosensor. MXenes have attracted considerable amount of interest due to their unique properties, morphology 
and important applications. With the excellent physical and chemical properties, such as graphene like structure, metallic conductivity, large electrochemically active surface, high stability, excellent mechanical properties and water solubility, MXene has been used in metal ion batteries, supercapacitors, fuel cells, electronic devices and absorbents [8-12]. This work indicates that the immobilization of proteins onto the MXene-based surfaces is a novel efficient method for the development of a new class of promising and sensitive nanostructured electrochemical biosensors with use in cancer diagnostics.

\section{MATERIALS AND METHODS}

\subsection{Materials}

Sarcosine oxidase from Bacillus sp. (25-50 U. $\left.\mathrm{mg}^{-1}\right)$, sarcosine, glutaraldehyde solution, chitosan, acetic acid, phosphate buffer $(\mathrm{PB})$ components $\left(\mathrm{KH}_{2} \mathrm{PO}_{4}\right.$ and $\mathrm{K}_{2} \mathrm{HPO}_{4}$ ) and Surine ${ }^{\mathrm{TM}}$ (a negative urine control for toxicology) were purchased from Sigma-Aldrich (USA). Micropolish alumina powder and polishing pads for disc electrodes were purchased from Buehler (USA). MXene was prepared using a previously described protocol [13].

\subsection{MXene-based sarcosine biosensor construction}

Prior to modification, all glassy carbon electrodes (GCEs) were mechanically polished by alumina powder, washed with distilled water and sonicated in ethanol and water successively. The biosensor was prepared by a simple drop-casting method. Firstly, chitosan solution $(0.1 \%)$ and $\mathrm{MXene}$ dispersion $\left(3 \mathrm{mg} \cdot \mathrm{ml}^{-1}\right)$ were mixed and shaken overnight at a temperature of $20{ }^{\circ} \mathrm{C}$ and at a rotation speed of $1500 \mathrm{rpm}$. After preparation, the above-prepared mixture was cast onto the surface of polished GCEs. After drying, $5 \%, 1 \%$ or no glutaraldehyde solution was applied to the surface for 10 minutes. Finally, SOx solution was applied onto the surface and allowed to dry at room temperature in a laminar box. Before electrochemical measurements, all the as-prepared electrodes were immersed in PB pH 7.4 to remove residual components. To optimise biosensor preparation and its performance, different configurations and nanocomposite preparation protocols were tested. Among all these configurations, from the stability point of view, only the configuration with mixed chitosan + MXene (overnight, $20^{\circ} \mathrm{C}, 1500 \mathrm{rpm}$ ) prior to drop-casting, subsequently modified by layer-by-layer using desalted and diluted $\mathrm{SO}$ with no GA activation, was chosen.

\subsection{Electrochemical measurements}

All electrochemical procedures were carried out on a laboratory potentiostat/galvanostat Autolab PGSTAT 302 $\mathrm{N}$ with an impedimetric module (Metrohm, Netherlands) with a GCE (Bioanalytical systems, USA) used as a working electrode. An Ag/AgCl/3 M KCl reference electrode and a counter platinum electrode (Bioanalytical systems, USA) were applied in a three-electrode cell system. Chronoamperometry measurements were performed on a rotating disc electrode employed as a working electrode (Metrohm, Netherlands). Cyclic voltammetry (CV) and chronoamperometry were performed in $0.1 \mathrm{M} \mathrm{PB} \mathrm{pH} 7.4$ purged with nitrogen for 15 minutes prior to use. $\mathrm{CV}$ measurements were run in the potential window from $0.1 \mathrm{~V}$ (volt) to $-1.0 \mathrm{~V}$ at a scan rate of $0.1 \mathrm{~V} \cdot \mathrm{s}^{-1}$. Chronoamperometry measurements were run at a fixed potential value of $-0.7 \mathrm{~V}$. All electrochemical measurements were run under Nova Software 1.10 (Metrohm, Netherlands) and data acquired were evaluated using Origin Pro 9.1 (Origin Lab, USA).

\subsection{Contact Angle Measurements}

Contact angle measurements for MXene-based interfaces were run on a portable instrument System E (Advex Instruments, Czech Republic) to obtain wetting angle and free surface energy. As the testing liquids, distilled water and diiodomethane were used. Free surface energy was determined using the two-liquid Owens-Wendt 
method. For each sample, the water and diiodomethane wetting angle was obtained as an average value of assays performed using 3 parallel measurements.

\subsection{Atomic force microscopy (AFM) and scanning electron microscopy (SEM)}

The morphology and structure of MXene and MXene-chitosan nanocomposite were characterized by scanning electron microscopy (SEM) and atomic force microscopy (AFM). SEM images of MXene and MXene-chitosan composite were recorded using Nova NanoSEM 450 microscope (FEI, USA) applying accelerating voltage. A peak force tapping mode AFM in air was carried out on a Bioscope Catalyst instrument and Olympus IX71 microscope (Bruker, USA) in conjunction with NanoScope 8.15 software.

\section{RESULTS AND DISCUSSION}

\subsection{Electrochemical measurements}

To evaluate the bioelectrochemical activity of the biosensor and to construct the corresponding calibration curves, the tested sarcosine concentration ranged from 2.5 to $50 \mu \mathrm{M}$ (Figure 1). Electrochemical reduction of $\mathrm{H}_{2} \mathrm{O}_{2}$ produced by catalytic decomposition of sarcosine by SOx present onto the surface of electrode started at $-0.4 \mathrm{~V}$ with the peak maximum present at $-0.7 \mathrm{~V}$. The cathodic peak for $\mathrm{H}_{2} \mathrm{O}_{2}$ reduction increased with the increasing biomarker concentration. Calibration curves were constructed by plotting sarcosine concentration against current response in the $1^{\text {st }} \mathrm{CV}$ scan after subtraction of a blank. Aiming to choose the best enzyme immobilization procedure an optimization of SOx immobilization was carried out using glutaraldehyde solution (Figure 1, inset).

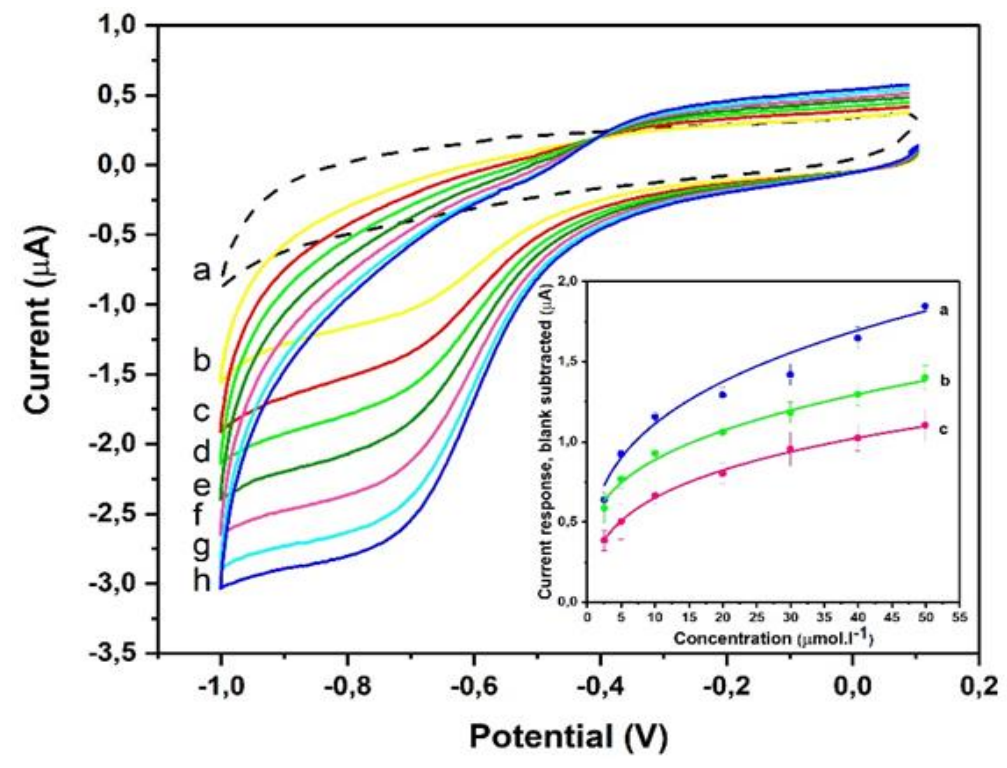

Figure 1 Cyclic voltammograms ( $\mathrm{CVs}$ ) of the prepared MXene-based biosensor in $\mathrm{N}_{2}$-purged $0.1 \mathrm{M} \mathrm{PB} \mathrm{pH}$ 7.4 containing 0 (a), 2.5 (b), 5 (c), 10 (d), 20 (e), 30 (f), 40 (g), 50 (h) $\mu \mathrm{M}$ sarcosine. Inset: Calibration plots obtained when using no glutaraldehyde (a), $1 \%$ glutaraldehyde (b) and $5 \%$ glutaraldehyde (c) during fabrication of the biosensor

However, the results obtained when using glutaraldehyde solution weren't satisfactory since glutaraldehyde application resulted in a decrease of a current responses. Calibration curves using a Hill model and MichaelisMenten kinetics renders the following maximal current outputs: $\left(I_{\max }\right):(2.61 \pm 0.15) \mu \mathrm{A}(0 \% \mathrm{GA}),(1.96 \pm 0.26)$ $\mu \mathrm{A}(1 \% \mathrm{GA})$, and $(1.42 \pm 0.10) \mu \mathrm{A}(5 \% \mathrm{GA})$. Glutaraldehyde solution has a negative effect on biosensor performance which can be explained by the formation a multi-point attachment of SOx to the surface, leading 
to enzyme inactivation. When no glutaraldehyde was present, the LOD of prepared SOx/MXene-chi/GCE biosensor (based on signal to noise ratio of 3) was calculated to be $91.4 \mathrm{nM}$ using $\mathrm{CV}$. Compared to the CV, chronoamperometry is more sensitive electrochemical method for indirect sarcosine detection. To obtain low LOD values, chronoamperometry on a rotating disc GCE at fixed potential of $-0.7 \mathrm{~V}$ and $900 \mathrm{rpm}$ was performed (Figure 2). The detection limit was calculated to be $18.0 \mathrm{nM}$ (based on a signal-to-noise ratio of 3$)$.

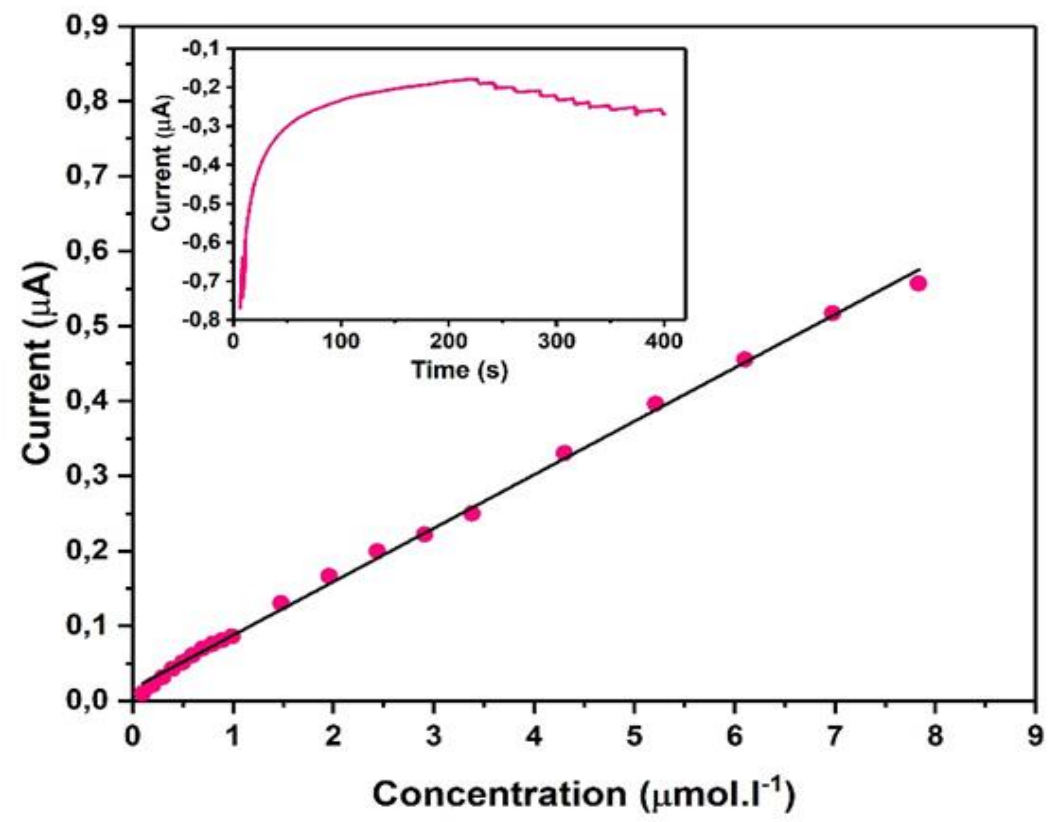

Figure 2 Plot of current responses after subtraction of blank of the prepared biosensor measured on a rotating disc GCE at $-0.7 \mathrm{~V}$ and $900 \mathrm{rpm}$ versus sarcosine concentration. Inset: a current versus time response of the prepared biosensor at $-0.7 \mathrm{~V}$ to successive addition of stock sarcosine solution in stirred $\mathrm{N}_{2}$ purged $0.1 \mathrm{M} \mathrm{PB} \mathrm{pH} 7.4$

\subsection{Contact Angle Measurements}

Wetting angle measurements were applied to investigate the surface modifications during individual steps of biosensor preparation. The hydrophilicity increased from bare GCE $\left(89.99 \pm 2.269^{\circ}\right)$, MXene-modified GCE $\left(71.87 \pm 4.968^{\circ}\right)$ and MXene/chitosan-modified GCE $\left(69.51 \pm 3.604^{\circ}\right)$. After SOx adsorption, the biosensor surface becomes more hydrophobic again $\left(87.44 \pm 4.898^{\circ}\right)$ (Figure 3 ).

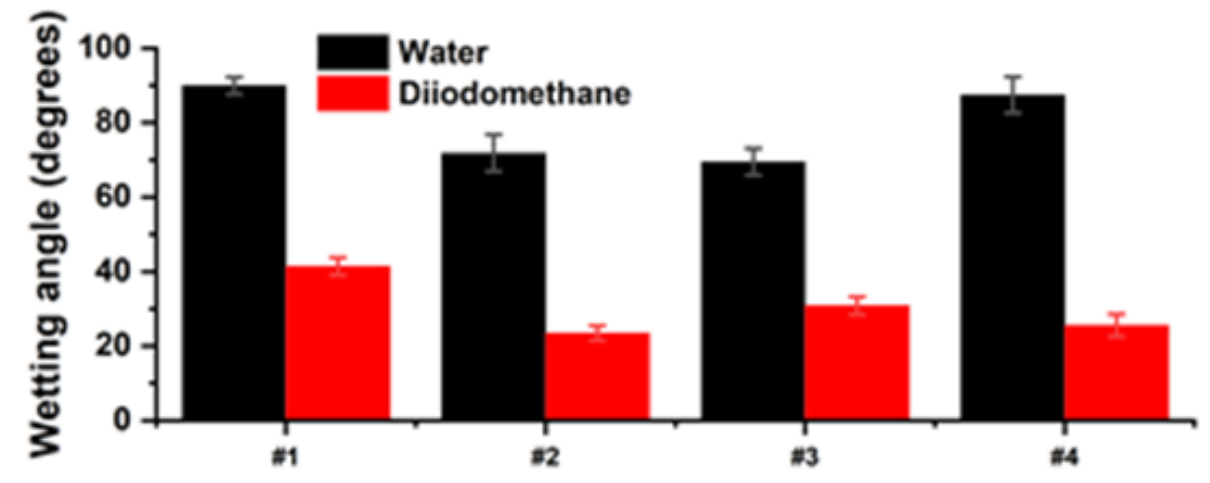

Figure 3 Graphical presentation of wetting angle measurements for: bare GCE (\#1), MXene-modified GCE (\#2), MXene/chitosan-modified GCE (\#3) and SOx-MXene/chitosan-modified GCE (\#4) 


\subsection{Attomic force microscopy (AFM) and scanning electron microscopy (SEM)}

SEM and AFM analysis was used to observe the microstructures of the samples and to investigate and see the surface morphology of prepared MXene and MXene-chitosan composite layers before enzyme immobilization took place. Studied MXene dispersion contains microscale-sized sheets with a multilayer nanostructure similar to exfoliated graphene oxide. Various sized MXene-chitosan composite aggregates with porous structure were also detected (Figure 4). Height profile analysis results revealed that MXene-chitosan flakes were about 10 times thicker than those consisting of MXene. Average thickness of one MXene nanosheet was calculated to be $(20.2 \pm 3.1) \mathrm{nm}$ (Figure 5).
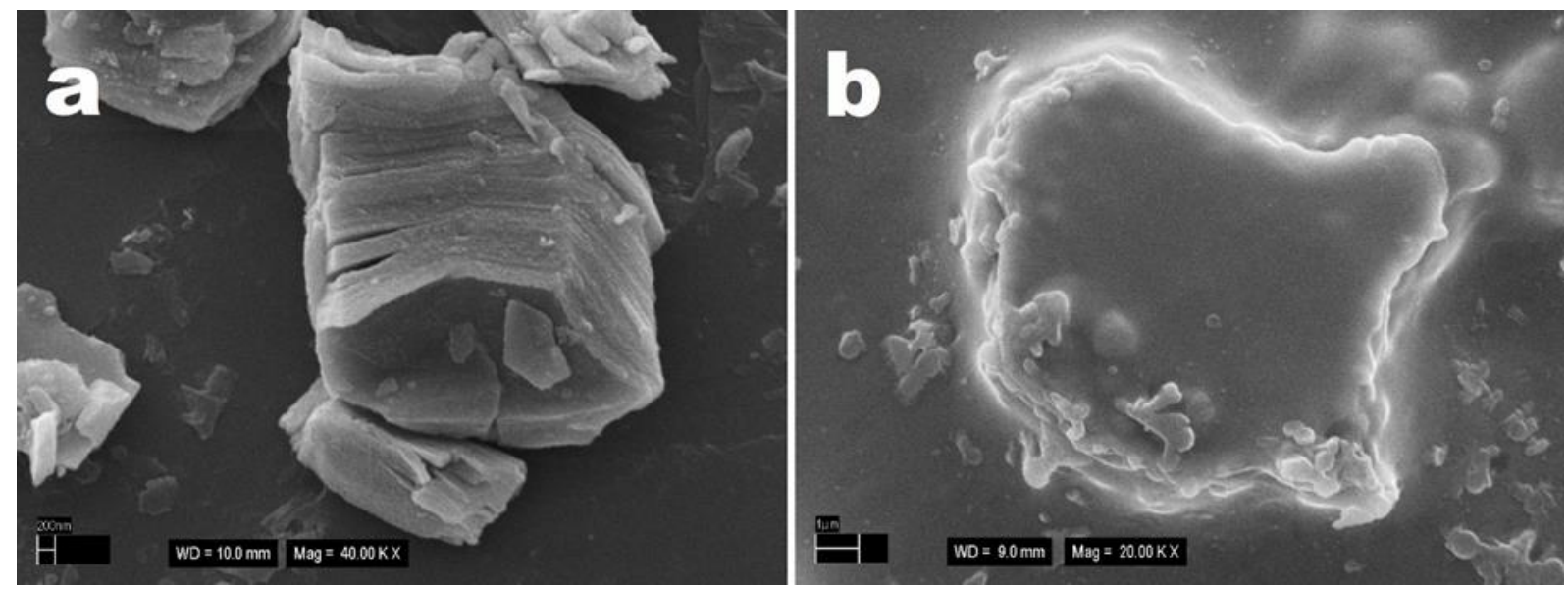

Figure 4 SEM images showing an unmodified $\mathrm{Ti}_{3} \mathrm{C}_{2} \mathrm{~T}_{x}$ MXene flakes, 40k magnification (a) and $\mathrm{Ti}_{3} \mathrm{C}_{2} \mathrm{~T}_{x}$ MXene/chitosan nanocomposite using 20k magnification (b)
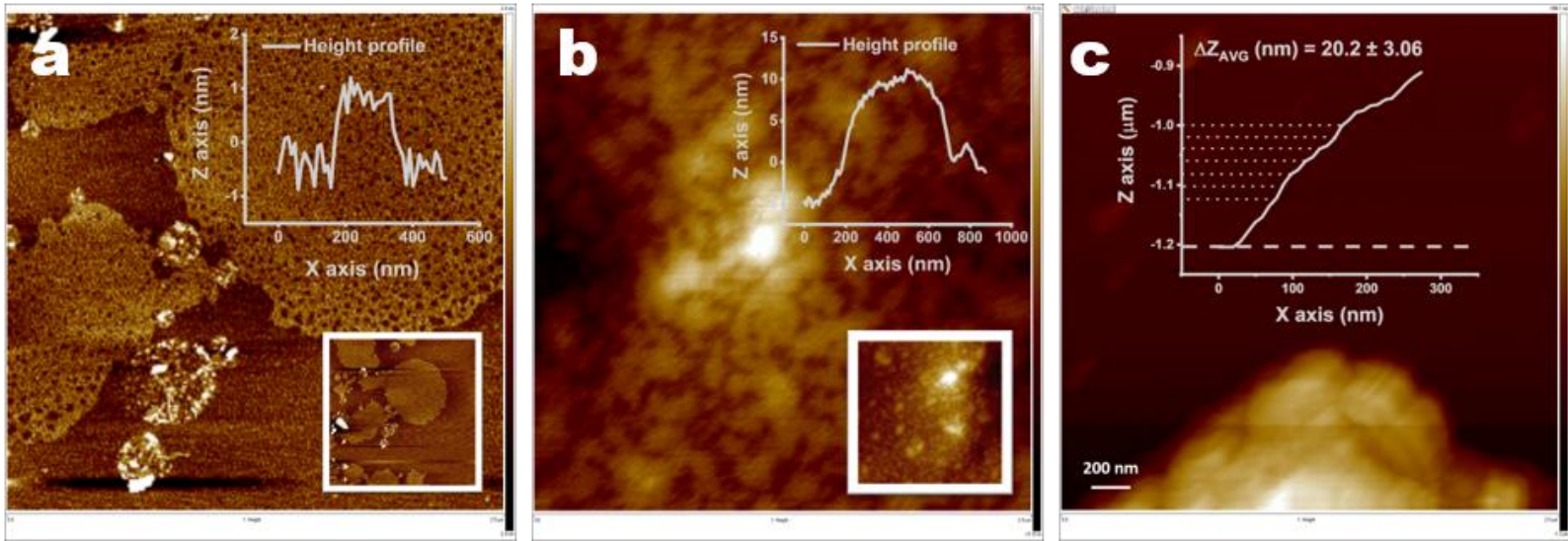

Figure 5 AFM images showing individual MXene sheets observed in MXene solution after a week of storage in an aqueous solution (a), individual MXene flake enwrapped in chitosan (b) and the edge of an unmodified MXene (c)

\section{CONCLUSION}

In this paper, a MXene-based biosensing platform was successfully fabricated for indirect sensitive detection of sarcosine. The sarcosine biosensor displayed low detection limit of $18 \mathrm{nM}$ and a linear range up to $7.8 \mu \mathrm{M}$ for sarcosine. The device was further used for sarcosine determination in artificial urine with a recovery index of $102.6 \%$. This work indicates that MXene is an excellent platform for protein-based biosensors applicable in medical diagnostics. 


\section{ACKNOWLEDGEMENTS}

\section{We would like to acknowledge the support received from the Slovak Research and Development Agency APVV 17-0300 and the support from the ERC Proof of Concept grant (No. 825586).}

\section{REFERENCES}

[1] Global burden of disease cancer collaboration. FITZMAURICE, C., ABATE, D., ABBASI, N. et. al. Global, Regional, and National Cancer Incidence, Mortality, Years of Life Lost, Years Lived with Disability, and DisabilityAdjusted Life-Years for 29 Cancer Groups, 1990 to 2017: A Systematic Analysis for the Global Burden of Disease Study. JAMA Oncology. [online]. 2019, vol. 5, no. 12, pp. 1749 - 1768. Available from: https://doi.org/10.1001/jamaoncol.2019.2996

[2] ABLIN, R. J., PIANA, R.: The Great Prostate Hoax: How Big Medicine Hijacked the PSA Test and Caused a Public Health Disaster. New York: St. Martin's Press, 2014.

[3] KATAFIGIOTI, A., KATAFIGIOTIS, I., SFOUNGARISTOS, S., et. al.: In the search of novel urine biomarkers for the early diagnosis of prostate cancer. Intracellular or secreted proteins as the target group? Where and how to search for possible biomarkers useful in the everyday clinical practice. Archivio Italiano di Urologia e Andrologia. [online]. 2016, vol. 88, no. 3, pp. 195 - 200. Available from: https://doi.org/10.4081/aiua.2016.3.195.

[4] JAKOBSEN, N. A., HAMDY, F. C., BRYANT, R. J.: Novel biomarkers for the detection of prostate cancer. Journal of Clinical Urology. [online]. 2016. Vol.9, pp. 3 - 10. Available from: https://doi.org/10.1177/2051415816656121.

[5] MARKIN, P. A., BRITO, A., MOSKALEVA, A., et. al.: Plasma Sarcosine Measured by Gas Chromatography-Mass Spectrometry Distinguishes Prostatic Intraepithelial Neoplasia and Prostate Cancer from Benign Prostate Hyperplasia. Laboratory Medicine. [online]. 2020, vol. 51, no. 6, pp. 566-573. Available from: https://doi.org/10.1093/labmed/Imaa008.

[6] SREEKUMAR, A., POISSON, L. M., RAJENDIRAN, T. M., et. al.: Metabolomic profiles delineate potential role for sarcosine in prostate cancer progression. Nature. [online]. 2009, vol. 457, no. 7231, pp. 910 -914. Available from: https://doi.org/10.1038/nature07762.

[7] HU, J., WEI, W., KE, S., et. al.: A novel and sensitive sarcosine biosensor based on organic electrochemical transistor. Electrochimica Acta. [online]. 2019, vol. 307, pp. 100 - 106. Available from: https://doi.org/10.1016/j.electacta.2019.03.180.

[8] DONG, Y., WU, Z. S., ZHENG, S., et. al.: $\mathrm{Ti}_{3} \mathrm{C}_{2}$ MXene-Derived Sodium/Potassium Titanate Nanoribbons for High-Performance Sodium/Potassium Ion Batteries with Enhanced Capacities. ACS Nano. [online]. 2017, vol. 11, no. 5, pp. 4792-4800. Available from: https://doi.org/10.1021/acsnano.7b01165.

[9] LUKATSKAYA, M. R., MASHTALIR, O., REN, C. E., et. al.: Cation intercalation and high volumetric capacitance of two-dimensional titanium carbide. Science. [online]. 2013, vol. 341, no. 6153, pp. 1502-1505. Available from: https://doi.org/10.1126/science.1241488.

[10] XIE, X., CHEN, S., DING, W., et. al.: An extraordinarily stable catalyst: Pt NPs supported on two-dimensional $\mathrm{Ti}_{3} \mathrm{C}_{2} \mathrm{X}_{2}(\mathrm{X}=\mathrm{OH}, \mathrm{F})$ nanosheets for oxygen reduction reaction. Chemical Communications. [online]. 2013, vol. 49, no. 86, pp. 10112-10114. Available from: https://doi.org/10.1039/c3cc44428g.

[11] MASHTALIR, O., NAGUIB, M., MOCHALIN, V. N., et. al.: Intercalation and delamination of layered carbides and carbonitrides. Nature Communications. [online]. 2013, vol. 4, art. no. 1716. Available from: https://doi.org/10.1038/ncomms2664.

[12] WANG, F., YANG, C., DUAN, M., et. al.: $\mathrm{TiO}_{2}$ nanoparticle modified organ-like $\mathrm{Ti}_{3} \mathrm{C}_{2}$ MXene nanocomposite encapsulating hemoglobin for a mediator-free biosensor with excellent performances. Biosensors and Bioelectronics. [online]. 2015, vol. 74, pp. 1022-1028. Available from: https://doi.org/10.1016/j.bios.2015.08.004.

[13] ALHABEB, M., MALESKI, K., ANASORI, B., et. al.: Guidelines for Synthesis and Processing of Two-Dimensional Titanium Carbide ( $\mathrm{Ti}_{3} \mathrm{C}_{2} \mathrm{~T}_{x} \mathrm{MXene}$ ). Chemistry of Materials. [online]. 2017, vol. 29, no. 18, pp. 7633 - 7644. Available from: https://doi.org/10.1021/acs.chemmater.7b02847. 